\title{
Gut microbiota metabolise food-derived mycotoxins
}

\author{
N. Daud, V.J. Currie, G. Duncan and S.W. Gratz \\ Rowett Institute, University of Aberdeen, Aberdeen, UK
}

Mycotoxins are fungal secondary metabolites which are frequently detected in agricultural crops including cereals. They are potent toxins and are important food contaminants which pose a potential risk to consumer health. In addition to fungal mycotoxins, plantderived mycotoxin metabolites, also called masked mycotoxins, are frequently found in cereals. These masked mycotoxins are sugarbound metabolites, and their contribution to overall toxicity in humans is less well understood. Hence, this study used a range of in-vitro model systems to predict the intestinal fate of masked mycotoxins in the human gut. The mycotoxins studied included the prevalent Fusarium mycotoxins deoxynivalenol (DON), nivalenol (NIV), T2-toxin (T2), HT2-toxin (HT2) and diacetoxyscripenol (DAS) and their respective glucose-bound metabolites DON-Glc, NIV-Glc, T2-Glc, HT2-Glc, DAS-Glc.

Artificial digestive juices (saliva, gastric juice, duodenal juice) were used to assess the stability of mycotoxins and masked mycotoxins in the small intestine. Human Caco-2 cells were used to study transport, metabolism and toxicity of mycotoxins ${ }^{(1)}$. Fully differentiated Caco-2 cultures were exposed to individual mycotoxins in culture medium in the apical compartment of Transwell filters and absorption and barrier disruption were assessed after 24 hours. Faecal batch cultures using human stool samples and single-strain incubations with important human gut strains were used to assess microbial metabolism of mycotoxins ${ }^{(2)}$. Individual mycotoxins were spiked into microbial cultures and incubated anaerobically for up to 48 hours. Mycotoxins were analysed using LC-MS/MS.

None of the mycotoxins or masked mycotoxins were metabolised by small intestinal juices and absorption studies showed that free mycotoxins are readily absorbed and can disrupt intestinal barrier function. In contrast, masked mycotoxins are not absorbed though the epithelial monolayer intact and can hence be transported to the large intestine. Upon contact with microbiota, masked mycotoxins were readily hydrolysed and free mycotoxins released into the culture supernatant. Marked differences were observed in the hydrolysis rates between different donor samples as well as between different masked mycotoxins treated with the same donor sample. Microbial de-acetylation was observed for several mycotoxin in all donor samples, whereas de-epoxydation was rare.

These results suggest that human gut microbiota are not efficient in detoxifying dietary mycotoxins. The rapid release of free mycotoxins from masked forms by human gut microbiota demonstrates that masked mycotoxins will contribute towards overall toxicity in humans and need to be included in risk assessments.

\section{Acknowledgements}

This work has been supported by the Scottish Government Rural and Environment Science and Analytical Services Division (RESAS) and a grant from Food Standards Agency (FSA).

\section{References}

1. Gratz SW, Dinesh R, Yoshinari T, et al. (2017) Mol Nutr Food Res 61 (4), 1-10.

2. Daud N, Currie V, Duncan G, Busman M \& Gratz S (2020) Int J Food Sci Nutr 71(5), 540-548. 